\title{
Charged Higgs Searches with D0
}

\author{
Phillip Gutierrez ${ }^{* \dagger}$ \\ University of Oklahoma \\ E-mail: pgutierrez@ou.edu
}

DØ has performed a number of searches for the charged Higgs boson in the mass range 80 to $300 \mathrm{GeV}$. For masses less than the top-quark mass two methods have been used: The ratio method where a deviation in the ratio of the $t \bar{t}$ cross section measured in the lepton + jets to dilepton channels is used to search for deviations from the standard model assumptions, and a global search where the correlations between the various top-quark cross section measurements are used to search for deviations in the standard model. For charged Higgs masses greater than the topquark mass, the direct search for an excess in single top-quark production in the invariant $t b$ mass is carried out. Preliminary and final results on these searches with $\approx 1 \mathrm{fb}^{-1}$ of data is presented.

Prospects for Charged Higgs Discovery at Colliders

16-19 September 2008

Uppsala, Sweden

\footnotetext{
${ }^{*}$ Speaker.

${ }^{\dagger}$ for the DØ Collaboration
} 


\section{Introduction}

The exact nature of the electroweak symmetry breaking sector of the standard model of particle physics (SM) remains an open question. At the present time, the SM relies on the Higgs mechanism [1] for the gauge invariant generation of particle masses through the use of a single SU(2) complex doublet. Three of the four fields in the complex doublet generate the masses of the gauge bosons $\left(Z, W^{ \pm}\right)$while the fourth leads to the as yet unobserved Higgs boson. Currently, there is no experimental data that limits the Higgs sector to a single complex doublet. Extensions to the SM Higgs sector that satisfy the requirements $\rho \equiv M_{W}^{2} / M_{Z}^{2} \cos ^{2} \theta_{W} \approx 1$, no flavor changing neutral currents (FCNC) at tree level, and that do not violate the unitarity bound are acceptable. This can be most naturally accomplished through the inclusion of additional Higgs doublets or Higgs singlets. The simplest such extension is one that adds to the Higgs sector a second complex SU(2) doublet; the two Higgs doublet model (2HDM). This additional complex doublet is also required in the minimal supersymmetric standard model (MSSM) in order for no anomalies to be present. Such a doublet introduces four additional particles, two neutral bosons and two charged bosons. In the analyses presented here, we search for evidence of such an extension to the SM by looking for evidence of the existence of a charged Higgs boson.

The Fermion couplings to the Higgs doublets are a priori not specified but are selected to avoid tree-level FCNC. The couplings can therefore be selected based on a theorem by Glashow and Weinberg [2], which states that if all fermions of a given electric charge couple to the same doublet, FCNC are absent at tree-level. This leads to two possibilities [3]: Type 1 models where all the fermions couple to a single doublet and Type 2 models where the up-type quarks and leptons couple to one doublet and the down-type quarks and leptons couple to the other doublet. The parameters of interest in Type 1 and 2 models are the mass of the charged Higgs $\left(M_{H^{ \pm}}\right)$and $\tan \beta$, the ratio of vacuum expectation values of the two Higgs doublets. An alternative to applying the Glashow and Weinberg theorem is to select the parameters of the model in such a manner that they suppress FCNC at tree-level and allow all quarks and leptons to couple to either or both Higgs doublets, which are called Type 3 models. In the large mass charged Higgs search, we use the Type 3 model described in Ref. [4] where FCNC are suppressed because of the small quark masses of the two lightest flavor generations.

We present $p \bar{p}$ collision data at $\sqrt{s}=1.96 \mathrm{GeV}$ collected using the $\mathrm{D} \varnothing$ detector [5] at the Fermilab Tevatron. The data samples correspond to $\approx 1 \mathrm{fb}^{-1}$ and are the same data samples as used in $t$-quark cross section analyses. Three separate charged Higgs searches are presented. The first two search for a charged Higgs with a mass less than the mass of the top-quark $M_{H^{ \pm}}<m_{t}$, while the third is a search for a charged Higgs with a mass greater than the top-quark mass $M_{H^{ \pm}}>m_{t}$.

\section{Ratio Method}

A powerful method of searching for deviations from the SM using the top-quark data is to measure the branching ratio times cross section for different decay channels, extract the $p \bar{p} \rightarrow t \bar{t}$ cross section $\left(\sigma_{t \bar{t}}\right)$ using the SM branching fractions, then compare the resulting cross sections. If the extracted cross sections are not the same, then one can interpret this as added $t$-quark decays to non-SM particles or as anomalous couplings. In this section we present such a search in $t$-quark 
decays under the assumption that any deviations observed are because of decays of the $t$-quark to charged Higgs $\left(t \rightarrow H^{+} b\right)$ [6].

We measure the $p \bar{p} \rightarrow t \bar{t}$ cross section through a variety of decay channels: $\ell^{ \pm}+$jets, $\ell^{+} \ell^{-}$, $\tau^{ \pm}+\ell^{\mp}$ and all jets where $\ell^{ \pm}$can be either an electron or muon. Since $B\left(t \rightarrow W^{+} b\right) \approx 100 \%$ in the SM, the branching fraction for each decay channel is determined by the $W$ decay branching fractions. If charged Higgs exists, this would no longer be the case and each decay channel would lead to a different measured value for $\sigma_{t \bar{t}}$ if only SM decays are assumed. For example, if charged Higgs exists, the ratio of measured cross sections $R_{\sigma}=\sigma_{t \bar{t}}^{\ell^{ \pm}}+$jets $/ \sigma_{t \bar{t}}^{\ell^{ \pm} \ell^{\mp}}$ would no longer be equal to one, but $R_{\sigma}$ would depend on the nature of the $H^{ \pm}$decay. We investigate a model where $B\left(H^{+} \rightarrow\right.$ $c \bar{s})=1$, which for $M_{H^{ \pm}}=80 \mathrm{GeV}$ has been demonstrated to lead to noticeable effects at the Tevatron for $\tan \beta \leq 3.5$ [7]. Such a leptophobic model can be realized in general multi-Higgsdoublet models (MHDM) [8]. In this model, the lepton plus jets channel contains events where one of the $t$-quarks decays to a charged Higgs, which is not possible in the dilepton channel. As shown in Fig. 1, the measured lepton plus jets cross section will be larger than the measured dilepton cross section, since the $H^{+} \rightarrow c \bar{s}$ decay mode adds to the number of lepton plus jet events, while subtracting from the number of dilepton events. Therefore, if a leptophobic charged Higgs exists, $R_{\sigma}>1$.

We have measured $\sigma_{t \bar{t}}$ in both the lepton plus jets [9] and dilepton [10] channels using data samples that correspond to $0.91 \mathrm{fb}^{-1}$ and $0.87 \mathrm{fb}^{-1}$ in $e^{ \pm}+$jets and $\mu^{ \pm}+$jets, respectively, and $1.04 \mathrm{fb}^{-1}, 1.05 \mathrm{fb}^{-1}$, and $1.05 \mathrm{fb}^{-1}$ in $e^{+} e^{-}, e^{ \pm} \mu^{\mp}$, and $\mu^{+} \mu^{-}$, respectively. The measured cross sections in the $\ell^{ \pm}+$jets channel with at least four jets is

$$
\sigma_{t \bar{t}}^{\ell^{ \pm}+\text {jets }}=8.27_{-0.95}^{+0.96}(\text { stat }+ \text { syst }) \pm 0.51 \text { (lumi) } \mathrm{pb}^{-1},
$$

and in the dilepton channel it is

$$
\sigma_{t \bar{t}}^{\ell^{ \pm} \ell^{\mp}}=6.8_{-1.1}^{+1.2}(\text { stat })_{-0.8}^{+0.9} \text { (syst) } \pm 0.4(\text { lumi }) \mathrm{pb}^{-1} \text {. }
$$

The $\geq 4$ jets requirement for the $\ell^{ \pm}+$jets cross section is used to limit the feed-through to a few percent of dilepton events into this channel.

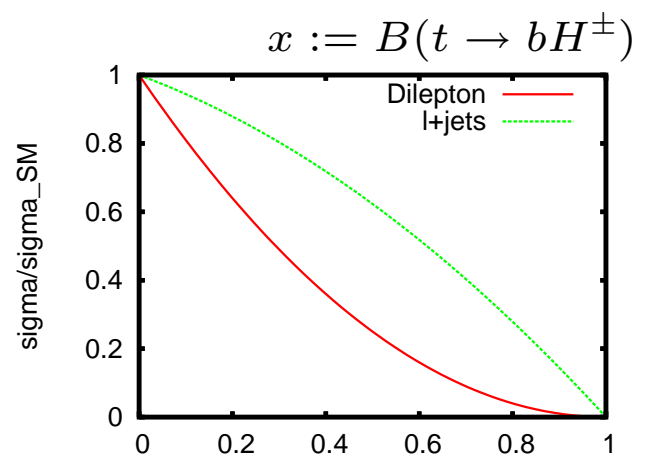

Figure 1: The modified cross sections relative to the SM cross section as functions of $B\left(t \rightarrow H^{+} b\right)$.

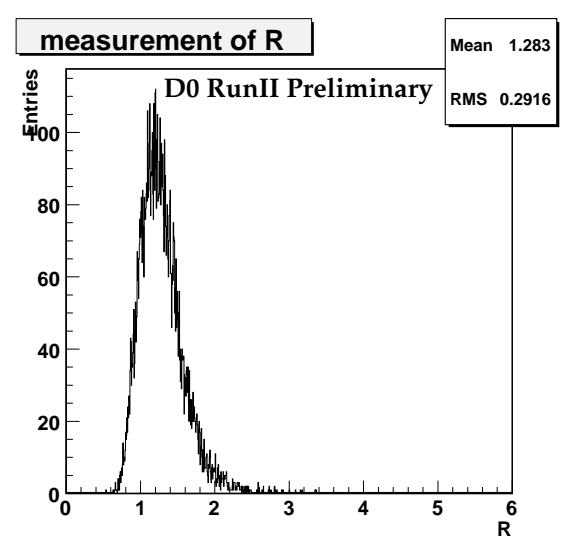

Figure 2: The cross section ratio, $R_{\sigma}$, distribution generated from the 10,000 pseudo-experiments. 
To extract the statistical and systematic uncertainties in the measurement of $R_{\sigma}$, we performed a series of pseudo-experiments that correctly include the correlations between the two measurements. In particular, those uncertainties that affect both channels, which include lepton and primary vertex identification, muon trigger, jet energy calibration, jet identification and resolution, and diboson background normalization are taken as $100 \%$ correlated. Those that affect only one of the two channels, are taken as uncorrelated. The uncertainties in the luminosity measurement are assumed to cancel. To account for statistical fluctuations, background and signal yields are sampled from a Poisson distribution using the measured central values. Each systematic uncertainty was modeled by smearing the central value of the cross section according to a Gaussian distribution of appropriate width. Figure 2 shows the result of 10,000 pseudo-experiments.

In order to calculate the confidence intervals of measured to generated cross section ratios, ensembles of $R_{\sigma}$ were generated by varying $\sigma_{t \bar{t}}^{\ell^{ \pm}}+$jets in steps of $0.5 \mathrm{pb}$ between 6 and $15 \mathrm{pb}$, and $\sigma_{t \bar{t}}^{\ell^{ \pm} \ell^{\mp}}$ fixed to its measured value. For each generated value of $R_{\sigma}, 10,000$ pseudo-experiments were performed with the resulting distribution fit to a Gamma function. After interpolating between the distributions for the different generated values of $R_{\sigma}$, we calculated continuous confidence intervals for generated $R_{\sigma}$ as a function of measured $R_{\sigma}$ using the method of Cousins and Feldman [11]. The confidence intervals are shown in Fig. 3. To determine the value of $R_{\sigma}$, we calculated the experimental ratio of the cross sections $R_{\sigma}^{\exp }=1.22$. This is then transformed to the true value along with the $68 \%$ confidence level interval as shown in Fig. 3. The result is

$$
R_{\sigma}=1.21_{-0.26}^{+0.27}(\text { stat }+ \text { syst })
$$

which is consistent with 1 .

Having measured $R_{\sigma}$, we use it to set limits on the existence of a leptophobic charged Higgs of $80 \mathrm{GeV}$ mass. Furthermore, we assume that the $t$-quark decays only to $W^{+} b$ and $H^{+} b ; B(t \rightarrow$ $\left.W^{+} b\right)+B\left(t \rightarrow H^{+} b\right)=1$. Accounting for the dilepton contribution to the $\ell^{ \pm}+$jets channel, the

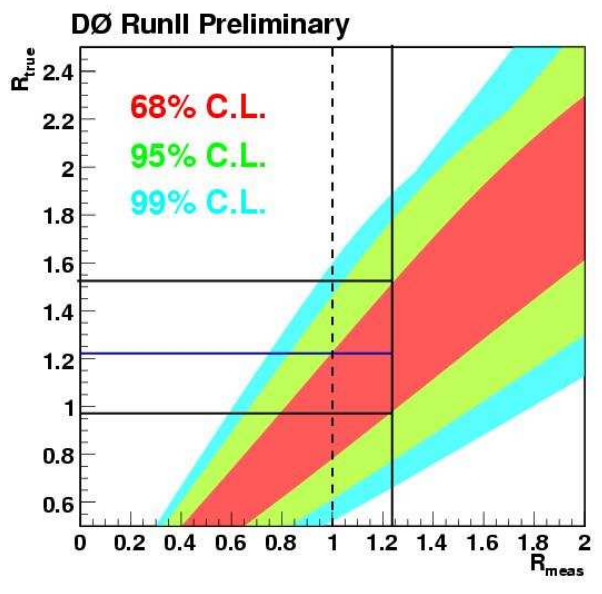

Figure 3: The Feldman-Cousins confidence interval bands as functions of measured and generated cross section ratio $R_{\sigma}$.

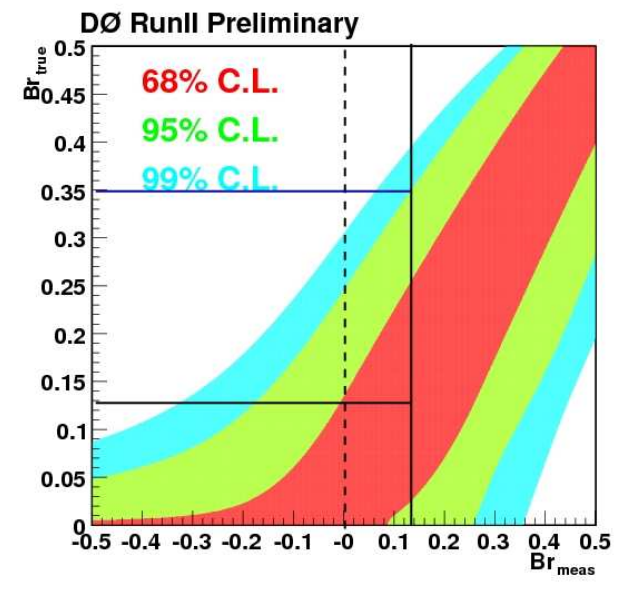

Figure 4: The Feldman-Cousins confidence interval bands as functions of measured and generated branching fraction $B\left(t \rightarrow H^{+} b\right)$. 
$t \rightarrow H^{+} b$ branching fraction can be expressed as follows:

$$
B \equiv B\left(t \rightarrow H^{+} b\right)=\frac{f \cdot\left(R_{\sigma}-1\right)}{1+f \cdot\left(R_{\sigma}-1\right)}
$$

where $f$ is a factor that accounts for the dilepton feed-through to the $\ell^{ \pm}+$jets channel.

Using a similar procedure as for the measurement of $R_{\sigma}$, we derive a limit on $B$ by performing a series of 10,000 pseudo-experiments varying the value of the measured $t$-quark cross section as measured in the $\ell^{ \pm}+$jets channel. Figure 4 shows the confidence intervals for generated $B$ as a function of measured $B$. Using Eq. 2.1 and Fig. 4, we arrive at the measured branching ratio $B=0.13_{-0.11}^{+0.12}$ (stat + syst). Since it is consistent with $B=0$, we proceed to set an upper limit, which was found to be $B<0.34$ at $95 \%$ confidence level (C.L.) with an expected value of $B<0.25$ at $95 \%$ C.L.

\section{Global Fit}

Combining the various $p \bar{p} \rightarrow t \bar{t}$ cross section measurements leads to increased sensitivity to non-SM physics. For example, under the assumption that $t \rightarrow H^{+} b$ is possible $\left(M_{H^{ \pm}}<m_{t}\right)$ and one considers purely tauonic decays of the charged Higgs, $B\left(H^{+} \rightarrow \tau^{+} v_{\tau}\right)=1$, the expected number of events for the lepton plus jets or dilepton channels will be less than predicted by the SM. But for $t$-quark decay modes that have $\tau$-leptons in the final state, the number of events will be larger than predicted by the SM. Therefore the correlation between measurements using the various channels increases our sensitivity to charged Higgs. Motivated by this rationale, we have carried out a search for charged Higgs using the $\ell^{ \pm}+$jets [9], dilepton [10], and $\tau^{ \pm}+\ell^{\mp}$ [12] channels in a global fit [13], where, as stated previously, $\ell^{ \pm}$refers only to $e^{ \pm}$and $\mu^{ \pm}$. These three basic channels are split into fourteen channels to improve the sensitivity: $8 \ell^{ \pm}+$jets channels with $e^{ \pm}$and $\mu^{ \pm}$and 3 or $>3$ jets and 1 or $>1 b$-tags; 4 dilepton channels with $e^{+} e^{-}, \mu^{+} \mu^{-}$, and $e^{ \pm} \mu^{\mp}$ plus 1 or $>1$ jets; $2 \tau^{ \pm}+\ell^{\mp}$ channels with $\ell^{ \pm}$either an $e^{ \pm}$or $\mu^{ \pm}$. The data samples for each of the fourteen channels are selected such that they are orthogonal to each other. This allows the channels to be combined using a simple product of the individual channel likelihoods.

In order to determine the expected number of events under various charged Higgs scenarios, we generated Monte Carlo (MC) samples of $p \bar{p} \rightarrow t \bar{t}$ events using PYTHIA [14] where one or both $t$-quarks decay to charged Higgs, $M_{H^{ \pm}}$is in the range of 80 to $155 \mathrm{GeV}$, and $m_{t}=170 \mathrm{GeV}$. Monte Carlo samples of SM $t$-quark decays were generated using the matrix element generator ALPGEN [15] and hadronized using PYHTIA. Using these MC samples along with background samples, the number of expected events (SM $t \bar{t}+$ background) for $\sigma_{t \bar{t}}=7.3 \pm 0.7 \mathrm{pb}$, the theoretical cross section at $m_{t}=172.6 \mathrm{GeV}$ (the world average $t$-quark mass), was found to be consistent with the number of observed events for all fourteen channels as shown in Fig. 5. Therefore, we proceeded to set limits on charged Higgs production.

The limits on $B\left(t \rightarrow H^{+} b\right)$ are calculated under two assumptions, the tauonic model $B\left(H^{+} \rightarrow\right.$ $\left.\tau^{+}+v_{\tau}\right)=1$ and the leptophobic model $B\left(H^{+} \rightarrow c \bar{s}\right)=1$, using the likelihood ordering prescription of Feldman and Cousins [11]. To account for statistical and systematic uncertainties, and correlations, ensembles of pseudo-experiments were generated. The result of the pseudoexperiments leads to the Feldman-Cousins confidence level bands of true branching ratio versus 

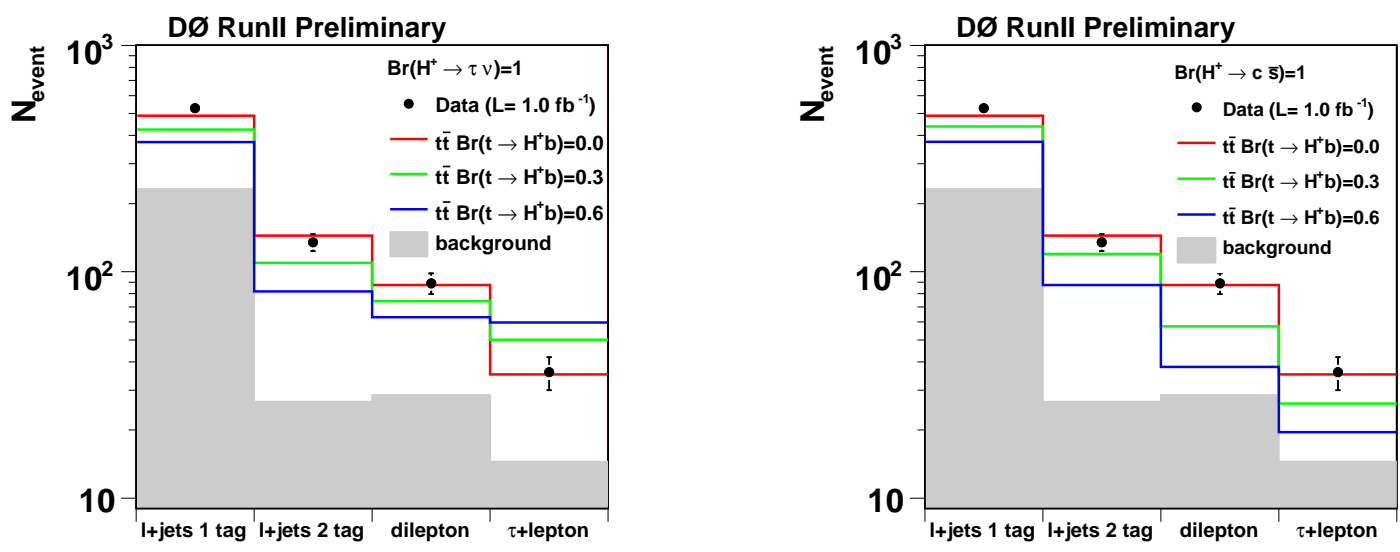

Figure 5: The observed data for different channels along with the predicated number of events for different values of $B\left(t \rightarrow H^{+} b\right)$ for the tauonic (left) and the leptophobic (right) models.

measured branching ratio. The confidence level bands are then converted into 95\% C.L. limits on the $B\left(t \rightarrow H^{+} b\right)$ versus $M_{H^{ \pm}}$using the measured value of the branching ratio. Figure 6 shows both the expected upper limit with the \pm 1 sigma band and the observed upper limit for the leptophobic, $B\left(H^{+} \rightarrow c \bar{s}\right)=1$, and tauonic, $B\left(H^{+} \rightarrow \tau \nu_{\tau}\right)=1$, models respectively.

A simultaneous fit to both $\sigma_{t \bar{t}}$ and $B\left(t \rightarrow H^{ \pm} b\right)$ is possible if the correlation between the two measurements is reasonably small. This is the case for the tauonic model, since there are channels that both increase and decrease the expected number of events. The leptophobic model has only channels that decrease the expected number of events, therefore a large correlation exists between $\sigma_{t \bar{t}}$ and $B\left(t \rightarrow H^{+} b\right)$. We therefore make a simultaneous measurement of $\sigma_{t \bar{t}}$ and $B\left(t \rightarrow H^{+} b\right)$ for the tauonic model, thereby making the measurement of the upper limit on $B\left(t \rightarrow H^{+} b\right)$ independent of the theoretical value of $\sigma_{t \bar{t}}$ and its associated uncertainties. The fitting procedure is the same as for the one dimensional fit and the limits are again extracted using the Feldman-Cousins method. The extracted value of $\sigma_{t \bar{t}}$ is found to be independent of $M_{H^{ \pm}}$. The upper limit on $B\left(t \rightarrow H^{+} b\right)$ is shown in Fig. 7, which gives a 30\% improvement for the lower values of $M_{H^{ \pm}}$over fitting for $B\left(t \rightarrow H^{+} b\right)$ using a fixed $\sigma_{t \bar{t}}$.
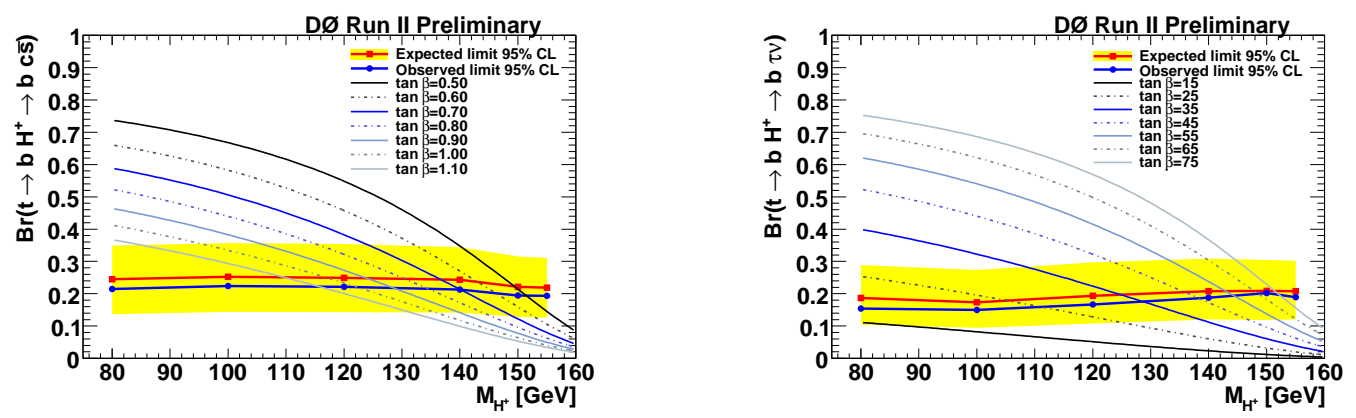

Figure 6: The observed (blue) and expected (red) limits with the one standard deviation band (yellow) for the leptophobic model (left) and tauonic model (right). Superimposed are the $2 \mathrm{HDM}$ prediction for constant $\tan \beta$. 
The upper limits on the branching ratio can be used to set exclusions bounds on the Type 2 $2 \mathrm{HDM}$ in regions of parameter space that correspond to either $B\left(H^{+} \rightarrow c \bar{s}\right)=1$ or $B\left(H^{+} \rightarrow\right.$ $\left.\tau^{+} v_{\tau}\right)=1$. Superimposed on the Figs. 6 to 7 are curves of constant $\tan \beta$ as functions of $M_{H^{ \pm}}$, which are used to convert the branching ratio to $\tan \beta$. The exclusion plot is shown in Fig. 8.

\section{High Mass Search}

Using $\approx 0.9 \mathrm{fb}^{-1}$ of data, we have carried out a search for direct $q \bar{q}^{\prime} \rightarrow H^{ \pm}$production of charged Higgs with mass greater than that of the $t$-quark [16]. In most models, a charged Higgs with $M_{H^{ \pm}}>m_{t}$ decays predominately to $t \bar{b}$ for values of $\tan \beta>1$ owing to the coupling constant being mass dependent and the large mass of the $t$-quark. The mass range for this search is restricted to masses greater than $180 \mathrm{GeV}$, which is approximately the minimum mass allowed for $H^{ \pm} \rightarrow t \bar{b}$ to be kinematically allowed, and less than $300 \mathrm{GeV}$ for which the cross section has decreased by an order of magnitude from its value at $180 \mathrm{GeV}$ in most models. This search complements previous searches at the Tevatron that have concentrated on $M_{H^{ \pm}}<m_{t}$.

The search for charged Higgs is carried out in the channel $p \bar{p} \rightarrow \ell^{ \pm}+E_{T}+$ jets, where the jets correspond to two $b$-jets. This is the same final state as used in the recently published "single top" analysis in the s-channel [17], therefore we use the same data sample for this analysis. The selection criteria are the same as in the "single top" analysis, except that we require exactly two jets in the event and one or two $b$-tags using the neural network $b$-tagger [18]. After all selections, the efficiency varies from about $0.5 \%$ to $1.25 \%$ with increasing $M_{H^{ \pm}}$.

Given the large charged Higgs mass, we use the $t \bar{b}$ invariant mass as the discriminating variable. In terms of the observables, this corresponds to the invariant mass of jet1 (the highest $p_{T}$ jet), jet2 (the second highest $p_{T}$ jet) and the $W$-boson. The $W$-boson 4-vector is reconstructed from the lepton 4-vector and the missing transverse energy $\left(\not_{T}\right)$. In most cases this leads to two solutions for the longitudinal momentum $\left(p_{z}\right)$ of the neutrino. The smallest absolute value of $p_{z}$ is selected. Figure 9 shows the $t \bar{b}$ invariant mass along with the expectations of a Type 3 2HDM.

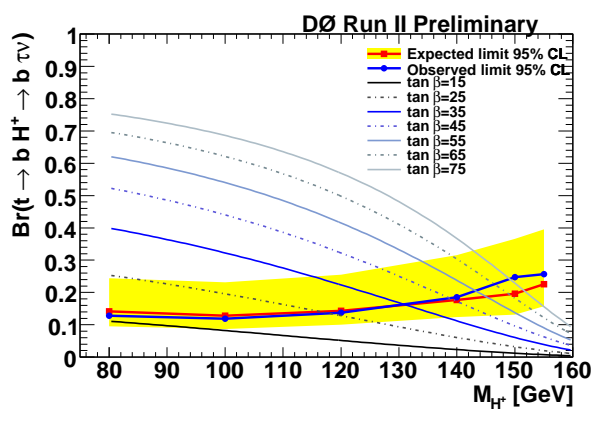

Figure 7: The observed (blue) and expected (red) limits with the one standard deviation band (yellow) for the tauonic model for the simultaneous fit to the cross section. Superimposed are the $2 \mathrm{HDM}$ prediction for constant $\tan \beta$.

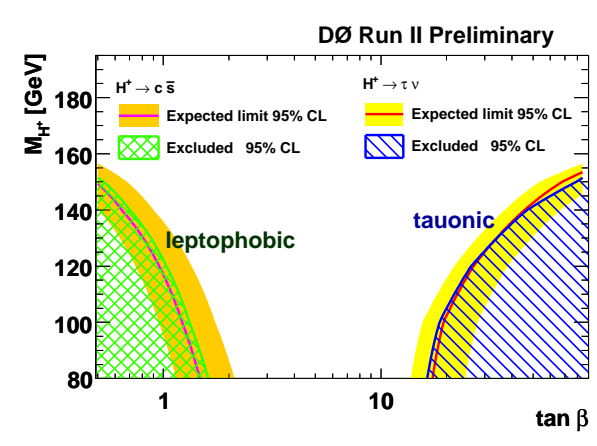

Figure 8: The observed (blue) and expected (red) limits with the one standard deviation band (yellow) in the $\left[M_{H^{ \pm}}, \tan \beta\right]$ plane for the $2 \mathrm{HDM}$. 


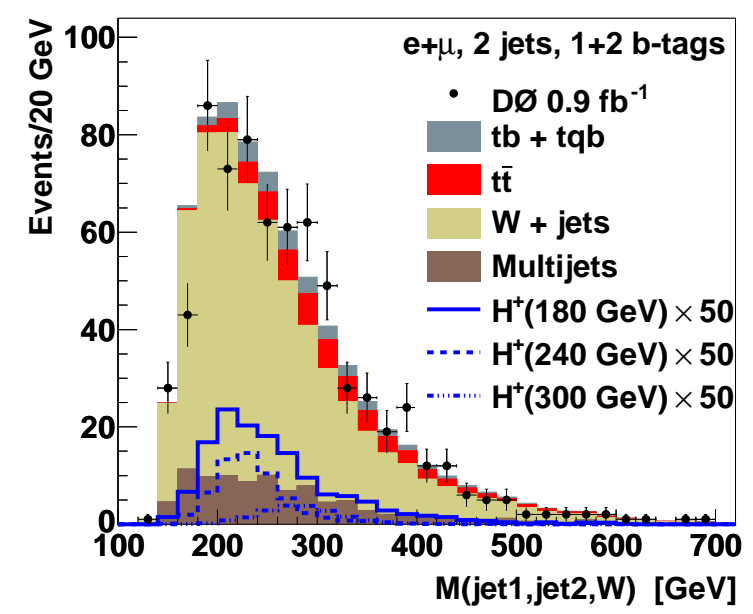

Figure 9: The distribution of the invariant mass $M($ jet1, jet2, $W)$, which is the discriminating variable in the $M_{H^{ \pm}}>m_{t}$ analysis. Shown are the expected signal and background, and the data. The signal corresponds to a Type 3 model.

Given that the data are consistent with the SM expectations, we proceeded to set upper limits on charged Higgs production. The limits are set for the Type 1, 2 and $32 \mathrm{HDMs}$ discussed earlier. In the $2 \mathrm{HDM}$, the effective Lagrangian for $H^{ \pm}$quark couplings is given by

$$
\mathscr{L}=\frac{g_{w}}{2 \sqrt{2}} H^{+} \bar{f}_{i}\left[g_{L}^{i j}\left(1-\gamma^{5}\right)+g_{R}^{i j}\left(1+\gamma^{5}\right)\right] f_{j}
$$

which is the sum of a left- and a right-handed chiral term with fraction determined by the coupling constants $g_{L}^{i j}$ and $g_{R}^{i j}$. Therefore to model charged Higgs production, we generate pure chiral state samples using the COMPHEP program [19]. The pure chiral state samples are then combined together according to the left- and right-handed couplings to simulate the desired version of the 2HDM. Table 1 gives the weights for the different models.

\begin{tabular}{|c|c|c|c|c|c|}
\hline & & $\tan \beta<0.1$ & $\tan \beta=1$ & $\tan \beta=5$ & $\tan \beta>10$ \\
\hline \multirow{2}{*}{$2 \mathrm{HDM}(\mathrm{I})$} & Left & 0.5 & 0.5 & 0.5 & 0.5 \\
\hline & Right & 0.5 & 0.5 & 0.5 & 0.5 \\
\hline \multirow{2}{*}{ 2HDM (II) } & Left & 0.99999 & 0.5 & 0.002 & 0.00001 \\
\hline & Right & 0.00001 & 0.5 & 0.998 & 0.99999 \\
\hline 2HDM (III) & $\begin{array}{l}\text { Left } \\
\text { Right }\end{array}$ & $\begin{array}{l}0.0 \\
1.0\end{array}$ & & & \\
\hline
\end{tabular}

Table 1: Event weights for combining left- and right-handed COMPHEP generated events. The Type 3 2HDM does not depend on $\tan \beta$.

The limits on $\sigma \times B$ are set constructing a binned likelihood function and using Bayesian techniques. A flat prior is used for signal cross section. All systematics and their correlations are taken into account. The limits are shown in Fig. 10, which are larger than the expectation from the three $2 \mathrm{HDM}$ models except for the Type 1 model with values of $M_{H^{ \pm}}$close to $180 \mathrm{GeV}$. This is 

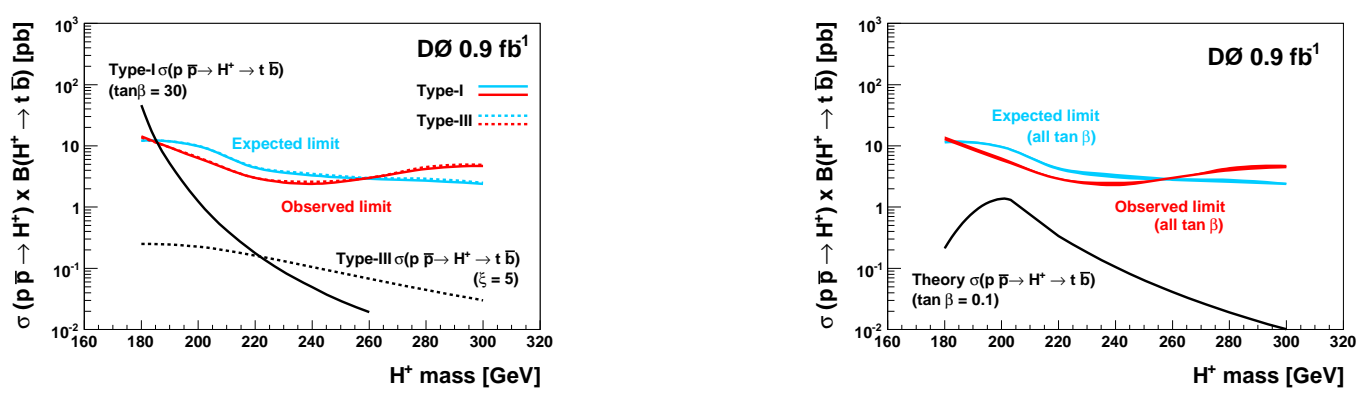

Figure 10: The expected (blue) and observed (red) limits on the cross section for $p \bar{p} \rightarrow H^{+} \rightarrow t \bar{b}$ for the Type 1 and $32 \mathrm{HDM}$ (left) and the Type 2 2HDM (right). Also shown is the theoretical cross section for such a model using $\tan \beta=0.1$.

used to exclude regions in the $\left[M_{H^{ \pm}}, \tan \beta\right]$ plane, see Fig. 11 . Regions in the $\left[M_{H^{ \pm}}, \tan \beta\right]$ plane that correspond to values of $\Gamma\left(H^{+} \rightarrow t \bar{b}\right)$ that are significantly greater than the detector resolution are not valid in this analysis.

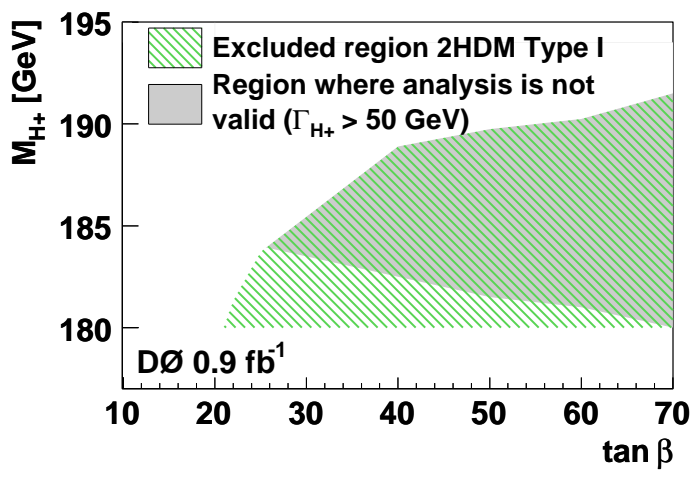

Figure 11: The 95\% C.L. excluded region in the $\left[M_{H^{ \pm}}, \tan \beta\right]$ plane for a type $12 \mathrm{HDM}$. The region with $\Gamma\left(H^{+} \rightarrow t \bar{b}\right)>50 \mathrm{GeV}$ corresponds to the region where the charged Higgs width is significantly larger than the detector resolution, therefore the analysis is not valid.

\section{Summary}

We have carried out three searches for charged Higgs bosons using the DØ detector. These searches cover the mass range from $80 \mathrm{GeV}$ up to $300 \mathrm{GeV}$. The searches for $H^{ \pm}$with $M_{H^{ \pm}}<$ $m_{t}$ use the $t$-quark production cross section measurements as their starting points and then look for deviations from expectations. One of the methods that we use compares the cross section measurements in two separate channels, where a deviation in the extracted values is because the SM branching ratios do not fully describe all the decay modes. We assume that any deviation is due to charged Higgs. The other method uses a global fit to the cross section measurements in the various channels by taking the correlations between all the measurements into account and again use this to search for indications of non-SM processes. Any deviation from SM predictions 
is interpreted as coming from charged Higgs. Neither of these methods found any deviation, and were therefore used to set limits on the existence of charged Higgs.

For $M_{H^{ \pm}}>m_{t}$, the method used the large invariant mass of the charged Higgs. In this case, the charged Higgs primary decay mode is to $t \bar{b}$, which is the same final state as the "single top" analysis in the $s$-channel. Therefore, we used the $t \bar{b}$ invariant mass as a discriminating variable for this search. Finding no evidence, we proceeded to set limits. For the Type 1 model we were also able to set exclusion bounds in the $\left[M_{H^{ \pm}}, \tan \beta\right]$ parameter space.

\section{References}

[1] P.W. Higgs, Phys. Lett. 12, 132 (1964); Phys. Rev. Lett. 13, 508 (1964); Phys. Rev. 145, 1156 (1966); F. Englert and R. Brout, Phys. Rev. Lett. 13321 (1964); Phys. Rev. Lett. 13321 (1964); G.S. Guralnik, C.R. Hagen and T.W.B. Kibble, Phys. Rev. Lett. 13585 (1964).

[2] S. Glashow and S. Weinberg, Phys. Rev. D 151958 (1977).

[3] J.F. Gunion, H.E. Haber, G. Kane, and S. Dawson, The Higgs Hunter's Guide, Frontiers in Physics (Addison-Wesley, Redwood City, Calif. 1990).

[4] H.-J. He and C.-P. Yuan, Phys. Rev. Lett. 83, 28 (1999).

[5] V.M. Abazov et al. (D0 Collaboration), Nucl. Instrum. Methods A 565, 463 (2006).

[6] The notation $H^{+}$refers to both $H^{+}$and its charge conjugate $H^{-}$. In a similar manner, the notation $q \bar{q}^{\prime}$ is used to represent both the $q \bar{q}^{\prime}$ and its charge conjugate $\bar{q} q^{\prime}$.

[7] A.G. Akeroyd, "Hidden Top Quark Decays to Charged Higgs Scalars at the Tevatron", hep-ph/9509203.

[8] Y. Grossman Nucl. Phys. B 425, 355 (1994).

[9] V.M. Abazov et al. (D0 Collaboration), Phys. Rev. Lett. 100, 192003 (2008).

[10] The DØ Collaboration, "Measurement of the $t \bar{t}$ Production Cross Section at $\sqrt{s}=1.96 \mathrm{TeV}$ in the Dilepton Final States Using $1 \mathrm{fb}^{-1}$,, DØ note 5371-CONF.

[11] G. Feldman and R. Cousins, Phys. Rev. D 57, 3873 (1998).

[12] The D $\emptyset$ Collaboration, "Measurement of $t \bar{t}$ Production Cross-Section in Lepton + Tau + b-jet(s) + Missing Transverse Energy Using $1 \mathrm{fb}^{-1}$ of Run II Data", DØ note 5451-CONF

[13] The DØ Collaboration, "A Search for Charged Higgs in $t \bar{t}$ Events”, DØ note 5715-CONF.

[14] T Sjöstrand et al., arXiv:hep-ph/0308153.

[15] M.L. Mangano et al., J. High Energy Phys. 0307, 001 (2003).

[16] V.M. Abazov et al. (D0 Collaboration), submitted to Phys. Rev. Lett. arXiv:0807.0859v1.

[17] V.M. Abazov et al. (D0 Collaboration), Phys. Rev. Lett. 98, 181802 (2007); V.M. Abazov et al. (D0 Collaboration), Phys. Rev. D 78, 012005 (2008).

[18] T. Scanlon, Ph.D. thesis, University of London, 2006, FERMILAB-THESIS-2006-43.

[19] E. Boos et al., (COMPHEP Collaboration), Nucl. Instrum. Methods A 534, 250 (2004). 